\title{
Design of Experiments for the Thermal Characterization of Metallic Foam
}

\author{
by \\ Paul E. Crittenden \\ Department of Mathematics, University of Nebraska \\ Lincoln, NE 68588-0656 \\ and \\ Kevin D. Cole \\ Mechanical Engineering Dept., University of Nebraska \\ Lincoln, NE 68588-0656
}

November 20, 2003

\begin{abstract}
Metallic foams are being investigated for possible use in the thermal protection systems of reusable launch vehicles. As a result, the performance of these materials needs to be characterized over a wide range of temperatures and pressures. In this paper a radiation/conduction model is presented for heat transfer in metallic foams. Candidates for the optimal transient experiment to determine the intrinsic properties of the model are found by two methods. First, an optimality criterion is used to find an experiment to find all of the parameters using one heating event. Second, a pair of heating events is used to determine the parameters in which one heating event is optimal for finding the parameters related to conduction, while the other heating event is optimal for finding the parameters associated with radiation. Simulated data containing random noise was analyzed to determine the parameters using both methods. In all cases the parameter estimates could be improved by analyzing a larger data record than suggested by the optimality criterion.
\end{abstract}




\section{Nomenclature}

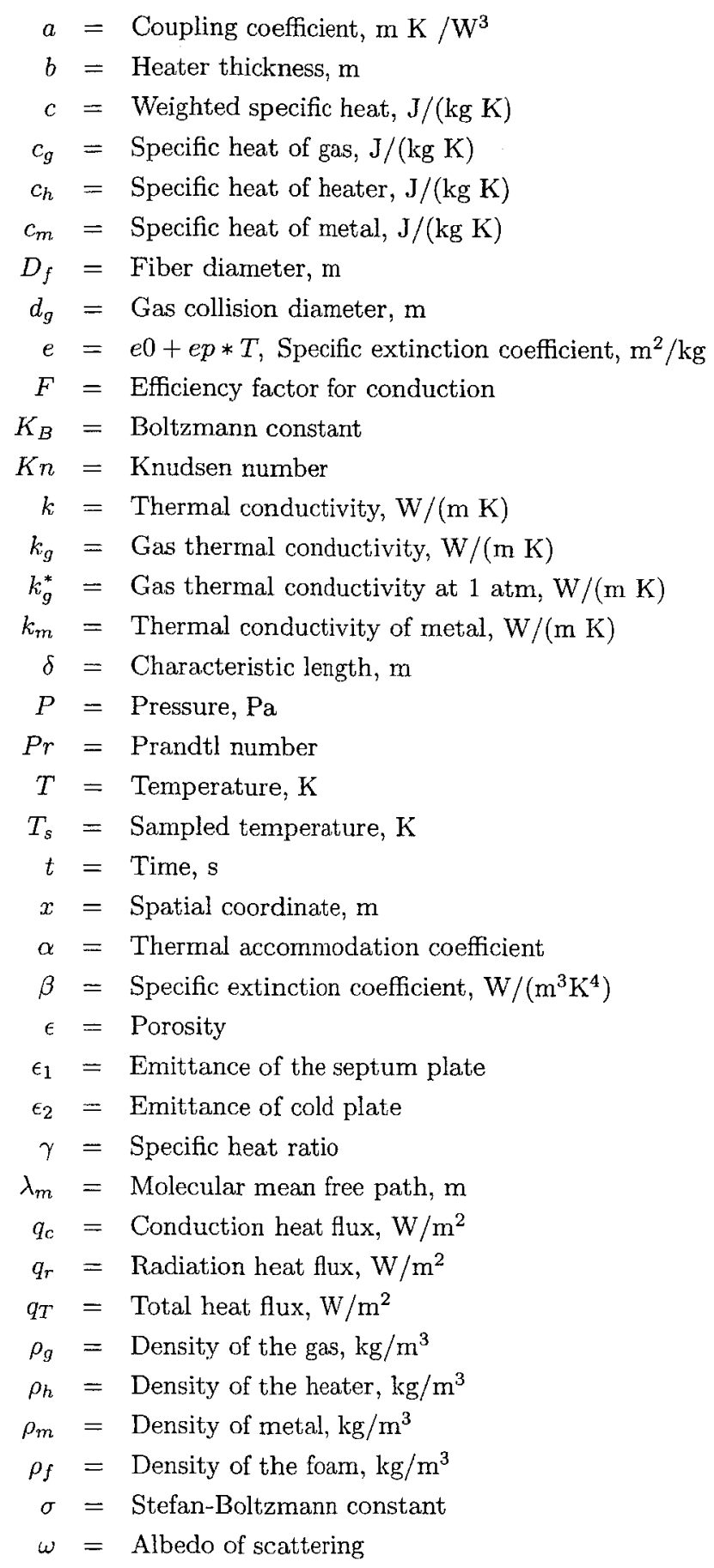




\section{Introduction}

High porosity metallic foams are being studied as possible components of aero-space thermal protection systems. The performance of these systems needs to be characterized over a wide range of temperatures and pressures during ascent and re-entry. ${ }^{1}$ In the future when such materials are specified as part of a vehicle program, part of the procurement process will involve certification that the material meets the specifications.

To date there has been little research on experiment design for thermal characterization of high-porosity materials. The present research is intended to close this gap in the procurement cycle by investigating transient methods for accurate measurement of thermal properties in metal-foam materials. In this paper only numerical simulations are presented.

A review of the pertinent literature is given next in the areas of optimal experiment design and heat transfer in high-porosity materials. Transient experiments combined with parameter estimation have been used for obtaining thermal properties for many years. ${ }^{2}$ In these methods the desired parameters are found by non-linear regression between the experimental data (temperatures in this case) and a computational model of the experiment. Parameter estimation concepts have recently been applied to optimal experiment design for thermal characterization of uniform materials ${ }^{3}$ and for materials with temperature-varying properties. ${ }^{4}$ One of us (Cole) has previously studied optimal experiment design for measurement of thermal conductivity in low-conductivity materials ${ }^{5}$ and in layered materials. ${ }^{6}$ The work was limited to a small rise in temperature so that radiation heat transfer was negligible. An optimality criterion was used to find the best experimental conditions for estimation of thermal properties.

There have been many studies of glass-fiber insulation in which combined radiation and conduction heat transfer are present. Yuen et. $\mathrm{al}^{9}{ }^{\mathrm{u}}$ used a detailed radiation model and measured optical properties to simulate the temperature response of a fibrous insulation material from first principles. The model involves a detailed determination of wavelength-dependent radiation exchange between multiple zones through the thickness of the material. Their results compared favorably to transient experimental tests carried out in 1974 on LI9000 sintered silica insulation (rigid space shuttle tile).

Insulation models based on optical properties, although science-based, do not provide direct evidence of the insulation's ability to withstand reentry heating. Simplified insulation models, combined with direct tests, can provide compelling evidence that a thermal-protection system is ready for use on a human-piloted vehicle. Daryabeigi ${ }^{7}$ used steady experiments to characterize low-density fibrous insulation over a wide temperature and pressure range and a transient experiment was used to simulate re-entry aerodynamic heating conditions. A combined radiation/conduction model was used, with the radiation described by a two-flux method assuming anisotropic scattering and a gray medium. Two models were discussed for combining solid and gas conduction into an effective conductivity, a parallel model and a model based on fiber orientation. Thermal properties estimated with both models yielded similar results, so it was not possible to choose one conduction model over the other. Low Rayleigh numbers and experimental verification ruled out free-convection heat transfer in the pores.

There arc fow studies of open-cell metallic foams. Zhu et. al ${ }^{10}$ carried out simulations of a titaninm foam material for the purpose of finding the minimum weight of the thermal protection by varying the pore size of the foam across its thickness. Their steady-state model included effective conductivity and radiation parameters. Compared to uniform materials, a density-graded material provides the same protection with less weight, with greater percentage savings for thinner insulation.

Sullins and Daryabeigi ${ }^{11}$ studied a Nickel-foam material over a wide range of temperatures and pressures with steady-state experiments combined with parameter estimation methods. The model for the heat transfer included a two-flux model for the radiation and combined gas/solid conduction. The effective conductivity was described by parallel gas-solid conduction, temperature-dependent gas conduction, and an efficiency factor for solid conductivity (as a small fraction of the bulk-metal conductivity). A gas-solid coupling term was also added to take into account an observed increase in conductivity at higher pressures and temperatures. The measurement of the model parameters was carried out with a non-linear regression technique, and the parameters reported.

In this paper the methods of optimal experiment design are applied to a high-porosity nickel foam material, with thermal property values taken from Sullins and Daryabeigi, ${ }^{11}$ Both conduction and radiation heat transfer are included. Based on a one-dimensional model of transient heat transfer, a large number 
of simulated experiments have been studied to determine which experimental conditions provide the best estimates of the thermal properties. No laboratory experiments are reported.

The paper is divided into several sections, as follows: analytical model; numerical solution; simulated experiments; optimality criterion; optimal experiment design; estimation of parameters; and conclusions.

\section{Analytical Model}

In this section the heat transfer model of Sullins and Daryabeigi ${ }^{11,12}$ is used to model metallic foams. It is a one dimensional, two-flux model taking into account both conduction and radiation heat transfer. Contained within the model are five parameters considered to be intrinsic properties of the material that must be determined empirically. Three of these intrinsic properties are related to the radiative heat transfer and two are related to heat transfer by conduction.

The transient heat equation with radiation is given by

$$
\rho c \frac{\partial T}{\partial t}=-\frac{\partial q_{c}^{\prime \prime}}{\partial x}-\frac{\partial q_{r}^{\prime \prime}}{\partial x}
$$

where $T$ is the temperature, $q_{c}^{\prime \prime}$ is the conduction heat flux, $q_{r}^{\prime \prime}$ is the radiant heat flux, $\rho$ is the density, and $c$ is the specific heat. The conduction heat flux is given by

$$
q_{c}^{\prime \prime}=-k \frac{\partial T}{\partial x}
$$

where $k$ is the thermal conductivity.

The gradient of the radiant heat flux is given by

$$
\frac{\partial q_{r}^{\prime \prime}}{\partial x}=\beta(1-\omega)\left(G-4 \sigma T^{4}\right)
$$

where $G$ is the incident radiation, $\omega$ is the albedo of scattering, $\beta$ is the extinction coefficient, and $\sigma$ is the Stefan-Boltzmann constant. The albedo of scattering is to be determined experimentally. The incident radiation is related to the radiant heat flux by

$$
q_{r}^{\prime \prime}=-\frac{1}{3 \beta} \frac{\partial G}{\partial x}
$$

Thus the heat equation (1) becomes

$$
\rho c \frac{\partial T}{\partial t}=\frac{\partial}{\partial x}\left(k \frac{\partial T}{\partial x}\right)+\frac{1}{3 \beta} \frac{\partial^{2} G}{\partial x^{2}} .
$$

The incident radiation, $G$, satisfies the second order differential equation

$$
-\frac{1}{3 \beta^{2}(1-\omega)} \frac{\partial^{2} G}{\partial x^{2}}+G=4 \sigma T^{4}
$$

on the interior. On the boundary, $G$ must satisfy:

$$
\begin{aligned}
& \frac{2}{3 \beta\left(\frac{\epsilon_{1}}{2-\epsilon_{1}}\right)} \frac{\partial G}{\partial x}+G=4 \sigma T_{h}^{4}, \quad x=0, \\
& \frac{2}{3 \beta\left(\frac{\epsilon_{2}}{2-\epsilon_{2}}\right)} \frac{\partial G}{\partial x}+G=4 \sigma T_{c}^{4}, \quad x=L,
\end{aligned}
$$

where $T_{h}$ is the temperature of the heater, $T_{c}$ is the temperature of the cold plate $(300.6 \mathrm{~K}), \epsilon_{1}=0.85$ is the emittance of the septum plate, and $\epsilon_{2}=0.92$ is the emittance of the cold plate. 
Next the material properties will be discussed for a specific high porosity nickel foam in a $N_{2}$ atmosphere. The product $\rho c$ is the weighted average of the values for the metal (nickel) and the gas (nitrogen). Explicitly,

$$
\rho c=\rho_{m} c_{m}(1-\epsilon)+\rho_{g} c_{g} \epsilon
$$

The density, $\rho_{g}$, and specific heat, $c_{g}$, of the gas are temperature dependent and given in the appendix. For nickel, $\rho_{m}$ is taken to be 8900 and $c_{m}=444.0$. The porosity, $\epsilon$, is 0.968 for nickel foam.

Heat conduction is assumed to occur in parallel in the gas and solid with some coupling in the form

$$
k=\epsilon k_{g}+(1-\epsilon) F k_{m}+a\left(F k_{g} k_{m}\right)^{2}
$$

where $F$ is the efficiency and $a$ is the coupling coefficient. The thermal conductivity of the metal is a function of temperature and is interpolated from tabulated data. ${ }^{13}$ For the gas

$$
k_{g}=\frac{k_{g}^{*}}{Z}
$$

where $k_{g}^{*}$ is the temperature dependent conductivity of the gas at atmospheric pressure (see Appendix) and $Z$ is given by

$$
Z=\Phi+2 \Psi \frac{2(2-\alpha) \gamma K n}{\alpha(\gamma+1) \operatorname{Pr}}
$$

In this expression, the thermal accommodation coefficient, $\alpha$, is taken to be one, $\gamma=1.4$ is the specific heat ratio, $\mathrm{Pr}$ is the temperature dependent Prandtl number (see Appendix), and $K n$ is the Knudson number. The Knudsen number is given by

$$
K n=\frac{\lambda}{\delta}
$$

where $\lambda$ is the gas molecular mean free path and $\delta$ is the pore size of the metallic foam. The quantities $\Phi$ and $\Psi$ were defined ${ }^{11}$ as

$$
\begin{gathered}
\Phi= \begin{cases}1, & K n<10 \\
0, & K n>10\end{cases} \\
\Psi= \begin{cases}0, & K n<.01 \\
1, & K n>.01\end{cases}
\end{gathered}
$$

This was to provide an approximation of $Z$ in the different ranges by neglecting one term when it was much smaller than the other. However, determining the optimum experiment requires finding the derivative of the temperature at the sensor with respect to the empirical parameters. Having a discontinuous expression for the gas conductivity resulted in inaccurate results for these derivatives when the simulated experiments were done near the boundary between two of the ranges for $K n$. Therefore in this work $\Phi=\Psi \equiv 1$.

The pore size for the metallic foam was assumed to be in the same form as given by Daryabeigi ${ }^{12}$ and is given by

$$
\delta=\frac{\pi}{4} \frac{D_{f}}{1-\epsilon} .
$$

In this expression $D_{f}=1.4 E-5 \mathrm{~m}$ is the diameter of the strut. The mean free path is given by

$$
\lambda=\frac{K_{B} T}{\sqrt{2} \pi P d_{g}^{2}}
$$

in which $K_{B}=1.38 E-23$ is the Boltzmann constant, $\mathrm{P}$ is the pressure, and $d_{g}=3.798 E-10 \mathrm{~m}$ is the gas collision diameter.

The extinction coefficient is given by

$$
\beta=e \cdot \rho
$$


where $\rho$ is the density of the foam and $e$ is the specific extinction coefficient. The specific extinction coefficient is taken to be a linear function of the temperature. Explicitly,

$$
e=e_{0}+e_{1} T
$$

The quantities $e_{0}$, and $e_{1}$ are considered to be intrinsic properties of the media to be determined experimentally.

Thus, for this model, heat transfer in a metallic foam is determined by the intrinsic properties $F, a$, $e_{0}, e_{1}$ and $\omega$. The goal is to find the optimal transient experiment to determine these parameters. The variables considered for different experiments are the heating power, sensor location, heating time and pressure.

\section{Numerical Solution}

The heat equation (5) and the radiation equation (6) are solved numerically. For each time step of the heat equation, the radiation equation (6) is discretized and solved. The solution is then used in a discretized form of the heat equation (5) to determine the temperatures for the next time step. The temperatures are then replaced with the new temperatures and the process is repeated.

Discretizing (6) through (8) gives the tri-diagonal system of equations

$$
\left[\begin{array}{ccccccc}
B_{1} & C_{1} & 0 & 0 & 0 & \ldots & 0 \\
A_{2} & B_{2} & C_{2} & 0 & 0 & \ldots & 0 \\
0 & A_{3} & B_{3} & C_{3} & 0 & \ldots & 0 \\
0 & 0 & \ddots & \ddots & \ddots & \ddots & 0 \\
0 & 0 & 0 & \ddots & \ddots & \ddots & 0 \\
0 & 0 & 0 & \ldots & A_{N-1} & B_{N-1} & C_{N-1} \\
0 & 0 & 0 & \ldots & 0 & A_{N} & B_{N}
\end{array}\right]\left[\begin{array}{c}
G_{1} \\
G_{2} \\
\vdots \\
\vdots \\
\vdots \\
\vdots \\
G_{N}
\end{array}\right]=\left[\begin{array}{c}
4 \sigma\left(T_{1}\right)^{4} \\
4 \sigma\left(T_{2}\right)^{4} \\
\vdots \\
\vdots \\
\vdots \\
\vdots \\
4 \sigma\left(T_{N}\right)^{4}
\end{array}\right]
$$

where $G_{i}$ and $T_{i}$ are the radiation and temperature, respectively, at node $i$. The elements of the coefficient matrix are given by

$$
\begin{aligned}
& A_{i}= \begin{cases}-\frac{2}{3 \beta_{i}^{2}(1-\omega) \Delta x_{i-1}\left(\Delta x_{i}-1+\Delta x_{i}\right)}, & i=2, \ldots, N-1, \\
-\frac{2\left(2-\epsilon_{2}\right.}{3 \beta_{i} \epsilon_{2} \Delta x_{i-1}}, & i=N,\end{cases} \\
& C_{i}= \begin{cases}-\frac{2\left(2-\epsilon_{1}\right)}{3 \beta \epsilon_{1} \Delta x_{i},}, 2 & i=1, \\
-\frac{2 \beta_{i}^{2}(1-\omega) \Delta x_{i}\left(\Delta x_{i-1}+\Delta x_{i}\right)}{3}, & i=2, \ldots, N-1,\end{cases} \\
& B_{i}= \begin{cases}1-C_{i}, & i=1, \\
1-C_{i}-A_{i}, & i=2, \ldots, N-1, \\
1-A_{i}, & i=N .\end{cases}
\end{aligned}
$$

In discretized form the heat equation (5) is

$$
\begin{aligned}
\left(\rho c_{j-1} \frac{\Delta x_{j-1}}{2}+\rho c_{j} \frac{\Delta x_{j}}{2}\right) \frac{T_{j}^{n+1}-T_{j}^{n}}{\Delta t} & =\frac{k_{j-1}}{\Delta x_{j-1}}\left(T_{j-1}^{n}-T_{j}^{n}\right)-\frac{k_{j}}{\Delta x_{j}}\left(T_{j}^{n}-T_{j+1}^{n}\right) \\
& +\frac{1}{3 \beta_{j-1} \Delta x_{j-1}}\left(G_{j-1}^{n}-G_{j}^{n}\right)-\frac{1}{3 \beta_{j} \Delta x_{j}}\left(G_{j}^{n}-G_{j+1}^{n}\right) .
\end{aligned}
$$

where superscripts have been added to $T$ and $G$ to indicate the time step. Solving for $T_{j}^{n+1}$ gives

$$
\begin{aligned}
T_{j}^{n+1} & =T_{j}^{n}-\frac{2 \Delta t}{\rho c_{j-1} \Delta x_{j-1}+\rho c_{j} \Delta x_{j}}\left(\frac{k_{j}}{\Delta x_{j}}\left(T_{j}^{n}-T_{j+1}^{n}\right)+\frac{1}{3 \beta_{j} \Delta x_{j}}\left(G_{j}^{n}-G_{j+1}^{n}\right)\right. \\
& \left.+-\frac{k_{j-1}}{\Delta x_{j-1}}\left(T_{j-1}^{n}-T_{j}^{n}\right)-\frac{1}{3 \beta_{j-1} \Delta x_{j-1}}\left(G_{j-1}^{n}-G_{j}^{n}\right)\right) .
\end{aligned}
$$


The material properties of the metallic foam, $\rho c, k$, and $\beta$, are all functions of temperature given by either polynomial approximations or interpolated from tabulated data. The temperature used for $\zeta_{i}=\rho_{i} c_{i}$, $k_{i}$, or $\beta_{i}$ is the average temperature of the two endpoints of the interval $\Delta x_{i}$. Explicitly for $\zeta=\zeta(T)$

$$
\zeta_{i}=\zeta\left(\frac{T_{i}+T_{i+1}}{2}\right)
$$

\section{Simulated Experiments}

In this section the simulated experiment for determining the thermal properties of the metallic foam is described. The simulated experiment consists of heating the (one-dimensional) sample of nickel foam, at some pressure, for a particular time period with a heater of a specified power, while taking temperature measurements at one location in the body. Thus the variables of the experiment are the heater power, the sensor location, the pressure, the heating time, and the total duration of the experiment.

Increasing the power to the heater increased the ability to determine the material properties. Since there would be physical limitations on the heater power supplied, rather than using the power of the heater as a variable between different experiments, an approximation of a heating apparatus used by Daryabeigi ${ }^{14}$ was made. This apparatus consisted of $2500 \mathrm{~W}$ cylindrical heating elements spaced $1.27 \mathrm{~cm}$ apart. This corresponds to about $310 \mathrm{MW} / \mathrm{m}^{2}$ and an effective thickness of $b=.00098 \mathrm{~m}$. The heater and the sample were placed $5.08 \mathrm{~cm}$ apart. A cold plate, kept at room temperature, is considered to be along the other side of the sample.

The temperature of the heater, node one in the discretized form of the heat equation, is determined from a lumped mass model. Under this model the heater must satisfy the differential equation

$$
\rho_{h} c_{h} b \frac{\partial T_{h}}{\partial t}=q_{i n}-q_{o u t}
$$

where $\rho_{h}=2600 \mathrm{~kg} / \mathrm{m}^{3}$ is the density of the heater and $c_{h}=800 \mathrm{~J} /(\mathrm{kg} \mathrm{K})$ is the specific heat of the heater. The power supplied to the heater is $q_{i n}=310 \mathrm{MW} / \mathrm{m}^{2}$ when the heater is powered and zero otherwise, while $q_{o u t}$ is the heat loss to the sample and the surroundings.

The losses from the heater include radiative transfer to the surroundings and to the sample. The radiative heat losses are given by

$$
q_{o u t}^{\tau}=\epsilon_{h} \sigma\left(T_{h}^{4}-T_{0}^{4}\right)+\epsilon_{1} \sigma\left(T_{h}^{4}-T_{s}^{4}\right)
$$

where $\epsilon_{h}=.05$ is the emittance of a water-cooled mirrored surface behind the heater, $T_{0}$ is the mirror temperature $(300.6 \mathrm{~K})$, and $T_{s}$ is the temperature of the surface of the sample. Gas conduction from the heater to the sample is also included, described by

$$
q_{o u t}^{c}=k_{g} \frac{\partial T}{\partial x}
$$

The total heat flow out of the heater is $q_{o u t}=q_{o u t}^{r}+q_{o u t}^{c}$.

A typical experiment starts at room temperature, then the heater is powered for a period of time while sampling the temperature at a particular location. Sampling continues for an additional period after the heater power is off. The heater model is incorporated into the transient finite difference code after the temperatures for each time step are found.

\section{$5 \quad$ Optimality Criterion}

The goal of this study is to examine a wide range of simulated experiments and determine the best experiment for determining the properties of the metallic foam. The suitability of individual experiments is determined from the sensitivity of the temperature with respect to the sought-after thermal properties of the sample, $F, a, e_{0}, e_{1}$ and $\omega$. Relabelling the parameters $b_{k}, k=1, \ldots, 5$, the normalized sensitivity coefficient for the $k$ th parameter, at the $i$ th time step, is defined as

$$
X_{k}(i)=b_{k} \frac{\partial T_{i}}{\partial b_{k}}
$$


Perturbing the parameters by a small amount, $(1+\delta) b_{k}$, the sensitivity coefficients can be approximated by

$$
X_{k}(i) \approx \frac{\left[T_{i}\left((1+\delta) b_{k}\right)-T_{i}\left(b_{k}\right)\right]}{\delta}, k=1, \ldots, 5
$$

The value of $\delta=.0001$ was found to give well-behaved values for $X$.

In general an experiment is better if the sensitivity coefficients are larger. In addition, for experiments with more than one parameter the sensitivity coefficients must be linearly independent. For these reasons the optimality criterion used is

$$
D=\frac{\left|\mathbf{X}^{T} \mathbf{X}\right|}{\left(n T_{\max }^{2}\right)^{p}}
$$

The sensitivity matrix, $\mathbf{X}$, is defined by

$$
\mathbf{X}^{T}=\left[b_{1} \frac{\partial \mathbf{T}}{\partial b_{1}}, b_{2} \frac{\partial \mathbf{T}}{\partial b_{1}}, \ldots, b_{5} \frac{\partial \mathbf{T}}{\partial b_{5}}\right]
$$

where $\mathbf{T}$ is a vector of the temperatures at each sampling time. In (32), $D$ is normalized by the maximum temperature, $T_{m a x}$, squared and the number of sample temperatures, $n$, all taken to the power $p$ (the number of parameters) in order to obtain a fair comparison among different experiments. ${ }^{3}$

The sensitivities are partial derivatives at a particular value of each parameter. The parameter values used for this study are given in Table 1, as reported by Sullins. ${ }^{11}$ For all of the examples the thickness of nickel foam was $13.6 \mathrm{~mm}$.

Table 1: Parameter values for simulated experiments

\begin{tabular}{ccccc}
\hline \hline $\mathrm{F}$ & $\mathrm{a}\left[(\mathrm{m} \mathrm{K} / \mathrm{W})^{3}\right]$ & $\omega$ & $e_{0}\left[\mathrm{~m}^{2} / \mathrm{kg}\right]$ & $e_{1}\left[\mathrm{~m}^{2} /(\mathrm{kg} \mathrm{K})\right]$ \\
\hline $6.85 \mathrm{E}-3$ & $3.89 \mathrm{E} 2$ & 9.85 & $-2.63 \mathrm{E}-3$ & $9.93 \mathrm{E}-1$ \\
\hline \hline
\end{tabular}

\section{Optimal Experiment Design}

Our experience with thermal conductivity measurements, with no radiation present, suggested that it is best to heat the sample as rapidly as possible for a period of time while taking measurements at the surface closest to the heater. This is also the best approach for radiation/conduction materials. A variety of simulated experiments were explored by varying the pressure, the duration of the heating and the total duration of the experiment. Initially the simulated experiments were terminated at the time corresponding to the peak value of the optimality criterion, $D$. It was found, however, that extending the experiment duration increased the accuracy of the parameter estimation. For experiments at lower pressures radiative heat transfer is more dominant, while at higher pressures heat transfer due to conduction is more significant. It was thus expected that the optimum case would occur at moderate pressures since three of the parameters are related to radiation and two are related to conduction.

The approximate conditions for the optimum experiment were found first by trial and error to find a rough value of the maximum of $D$, after which an optimization routine was run with the rough value as the initial guess. The maximum value of $\mathrm{D}$ found was $3.38 \mathrm{E}-16$. This value resulted from an experiment conducted at $0.190 \mathrm{~mm} \mathrm{Hg}$ in which the heater was run for 269 seconds and the temperature was sampled every 10 seconds for a total duration of 840 seconds. In Figure 1 the optimality criteria is graphed versus time for a few simulated experiments in which the optimum heating time was used and the pressure was varied around the optimal pressure. In Figure 2, D is graphed versus time with the pressure fixed at 0.19 $\mathrm{mm} \mathrm{Hg}$ for various heating times. As can be seen from the figure, the exact heating duration is not too critical. For heating times between 260 and 290 seconds the maximum value of $\mathrm{D}$ only varies by about four percent. In general if the sample is continuously heated the $\mathrm{D}$ value rises to a maximum and then begin to 
decrease as the system reaches steady state. If instead the heater is shut off, at some time, the the value of $\mathrm{D}$ dramatically increases until the system again nears steady state (room tempcrature). The rise in the $D$ values when the heater is shut off is so dramatic that in most cases it is impossible to see the rise in the $\mathrm{D}$ values during heating if the entire experiment is graphed on the same scale. In Figure 3 the same curves given in Figure 2 are repeated along with a curve corresponding to constant heating for 1200 seconds. The separation in the four curves prior to the heater being shut off is due to each experiment reaching a different maximum temperature which is included in the normalization. The best time for heating (269 seconds) appears to be near the location where the $\mathrm{D}$ curve changes from concave up to concave down. In Table 2 the maximum value of $\mathrm{D}$ obtained for various pressures and heating times is summarized.

Table 2: Maximum D values for experiments

\begin{tabular}{ccccccc}
\hline & \multicolumn{7}{c}{ Heating Duration [sec] } \\
\hline $\mathrm{P}[\mathrm{mmHg}]$ & 250 & 260 & 269 & 280 & 290 & 300 \\
\hline 0.09 & $1.30 \mathrm{E}-16$ & $1.30 \mathrm{E}-16$ & $1.30 \mathrm{E}-16$ & $1.30 \mathrm{E}-16$ & $1.28 \mathrm{E}-16$ & $1.27 \mathrm{E}-16$ \\
\hline 0.14 & $2.69 \mathrm{E}-16$ & $2.71 \mathrm{E}-16$ & $2.72 \mathrm{E}-16$ & $2.71 \mathrm{E}-16$ & $2.69 \mathrm{E}-16$ & $2.67 \mathrm{E}-16$ \\
\hline 0.19 & $3.19 \mathrm{E}-16$ & $3.23 \mathrm{E}-16$ & $3.38 \mathrm{E}-16$ & $3.25 \mathrm{E}-16$ & $3.24 \mathrm{E}-16$ & $3.22 \mathrm{E}-16$ \\
\hline 0.24 & $4.32 \mathrm{E}-16$ & $4.11 \mathrm{E}-16$ & $3.93 \mathrm{E}-16$ & $3.78 \mathrm{E}-16$ & $3.65 \mathrm{E}-16$ & $3.53 \mathrm{E}-16$ \\
\hline 0.29 & $4.73 \mathrm{E}-16$ & $4.51 \mathrm{E}-16$ & $4.32 \mathrm{E}-16$ & $4.15 \mathrm{E}-16$ & $4.01 \mathrm{E}-16$ & $3.89 \mathrm{E}-16$ \\
\hline \hline
\end{tabular}

It should be noted that for the optimal experiment one would want to stop taking data as soon as D reached its maximum value. From Figure 1 or Figure 2 this is at 840 seconds. This time is also not too critical, since for any value between 830 seconds and 900 seconds D will still be within one percent of its maximum value. Thus for this model the optimal experiment for determining the material properties is for the sample to be heated for 270 seconds at a pressure of $0.19 \mathrm{~mm} \mathrm{Hg}$ with sampling every 10 seconds until 840 seconds have elapsed.

In Figure 4 the heat flux for the optimal experiment is given as a function of time. The total heat flux is approximately a step function while the radiative heat flux and heat flux due to conduction are of the same order, as expected. The temperature profile for the optimal experiment is given in Figure 5. One can see from the temperature profile the system has not reached steady state when the heater is shut off.

\section{$7 \quad$ Estimation of Parameters}

In this section the parameters are determined using three simulated experiments. Experiment 1 is the experiment found using the optimality criterion in the previous section. Experiment 2 is the same as experiment 1 except data is taken for 2000 seconds. Experiment 3 consists of two heating events concatenated into one simulated experiment, where one of the heating events is dominated by conduction flux and the other is dominated by radiative flux. Data for all of the simulated experiments are obtained by calculating the exact temperatures using the values of the parameters given in Table 1 and then corrupting them with random noise with a standard deviation of $0.08 \mathrm{~K}$. The standard deviation was chosen to correspond with the maximum standard deviations reported by Sullins ${ }^{11}$ for actual experiments in the same temperature ranges. A nonlinear Levnberg-Marquardt parameter estimation routine is used to fit the model parameters. ${ }^{15}$

For simulated experiment 1 the optimum experiment determined in the previous section was used. It consists of running the heater for 269 seconds and taking measurements until 840 seconds elapsed. The gas pressure is $0.19 \mathrm{~mm} \mathrm{Hg}$. The residuals between the exact and perturbed (noise added) temperatures, $T_{\text {pert }}-T_{\text {exact }}$, and between the exact temperatures and those calculated using the estimated parameters, $T_{\text {est }}-T_{\text {exact }}$, are given in Figure 6 . The residuals between the exact temperatures and those calculated with the estimated parameters is less than $0.5 \mathrm{~K}$ and is biased towards overestimating the exact temperatures. In addition the error in the calculated temperatures exceeds the random noise added to the exact values. The estimated values of the parameters along with their $90 \%$ confidence intervals are given in Table 3 .

Since at 840 seconds the temperature was still significantly above room temperature, for simulated 
Table 3: Parameter values for simulated experiment 1

\begin{tabular}{cccccc}
\hline \hline & $e_{0}$ & $e_{1}$ & $\omega$ & $\mathrm{F}$ & $\mathrm{a}$ \\
\hline Exact & $9.850 \mathrm{E}+00$ & $-2.630 \mathrm{E}-03$ & $9.930 \mathrm{E}-01$ & $6.850 \mathrm{E}-03$ & $3.890 \mathrm{E}+02$ \\
\hline Estimated & $9.838 \mathrm{E}+00$ & $-2.619 \mathrm{E}-03$ & $9.930 \mathrm{E}-01$ & $6.582 \mathrm{E}-03$ & $4.696 \mathrm{E}+02$ \\
\hline Percent Error & -0.127 & $-4.17 \mathrm{E}-01$ & $4.38 \mathrm{E}-03$ & $-3.91 \mathrm{E} 00$ & $2.07 \mathrm{E} 01$ \\
\hline $90 \%$ Confidence Interval & $6.551 \mathrm{E}-03$ & $4.922 \mathrm{E}-06$ & $3.152 \mathrm{E}-05$ & $7.956 \mathrm{E}-05$ & $2.821 \mathrm{E}+01$ \\
\hline \hline
\end{tabular}

experiment 2 the same heating time was used, but the temperature sampling was extended to 2000 seconds. By 2000 seconds the temperature at the sensor had cooled to $305 \mathrm{~K}$. The results were much better both in terms of the residuals (See Figure 7) and the parameter estimation (see Table 4). The errors in the temperatures were less than $.05 \mathrm{~K}$ while the parameters were found accurately within about one percent.

Table 4: Parameter values for simulated experiment 2

\begin{tabular}{cccccc}
\hline \hline & & & & & \\
& $e_{0}$ & $e_{1}$ & $\omega$ & $\mathrm{F}$ & $\mathrm{a}$ \\
\hline Exact & $9.850 \mathrm{E}+00$ & $-2.630 \mathrm{E}-03$ & $9.930 \mathrm{E}-01$ & $6.850 \mathrm{E}-03$ & $3.890 \mathrm{E}+02$ \\
\hline Estimated & $9.853 \mathrm{E}+00$ & $-2.633 \mathrm{E}-03$ & $9.930 \mathrm{E}-01$ & $6.874 \mathrm{E}-03$ & $3.819 \mathrm{E}+02$ \\
\hline Percent Error & $3.489 \mathrm{E}-02$ & $1.225 \mathrm{E}-01$ & $-1.233 \mathrm{E}-04$ & $3.521 \mathrm{E}-01$ & $-1.824 \mathrm{E}+00$ \\
\hline $90 \%$ Confidence Interval & $6.569 \mathrm{E}-03$ & $4.925 \mathrm{E}-06$ & $3.191 \mathrm{E}-05$ & $5.118 \mathrm{E}-05$ & $1.529 \mathrm{E}+01$ \\
\hline \hline
\end{tabular}

Table 4 shows that simulated experiment 2 estimated all of the parameters accurately, except for the coupling coefficient, $a$. Various other experiments with single heating events were tried, without success, in order to find the coupling coefficient more accurately. The coupling coefficient is only significant when the conduction through the gas is significant. This means a will be more important in experiments conducted at high pressure. In addition it should be easier to determine $a$ from an experiment in which the radiative heat transfer is less important. Based on this information, simulated experiment 3 was constructed from a pair of heating events: one that was optimal for determining the conduction parameters; and one that was optimal for finding the radiation parameters. Noise was added to the data from this pair of heating events. The data sets were then concatenated as one data set for the parameter estimation. It should be noted that although optimal heating events were found for both the conduction and radiation parameters with the other parameters fixed, for the analysis of the concatenated data, all of the parameters were considered to be unknown.

For the conduction-optimal heating event, increasing pressure was found to improve the estimation of $a$ and $F$. However, an increase in pressure from 1 to 10 atmospheres increased the $D$ values by less than one percent. Since it would be much easier to obtain a pressure of one atmosphere this was used for the conduction-optimum heating event. In this case, it was also found that using more power in the heater was better. Therefore the only variables to determine was how long to power the heater and how long to sample the temperature. From Figure 4 it can be seen that the flux due to conduction reaches a peak at about 20 seconds. At one atmosphere this peak moves to about 23.6 seconds, which was used for the conduction-optimal heating event. The temperature was sampled every second instead of every ten seconds. It was found that a data duration of 150 seconds provided good estimation of conduction parameters. Strict use of the $D$ parameter would have resulted in a shorter experiment, but as with the single heating event the sample had not cooled very close to steady state by that time.

For the radiation-optimal heating event, $D$ is the largest for a vacuum (no gas conduction). Sullins ${ }^{11}$ reported using a minimum pressure of $0.0001 \mathrm{~mm} \mathrm{Hg}$ so this value was chosen as a practically obtainable minimum pressure. For the experiments discussed so far, the initial heating had a significant conduction flux (see Figure 4). In Figure 8, the ratio of the conduction heat flux to the radiation heat flux is plotted for a few different heating times. It can be seen from the figure that if the sample is heated for 30 seconds this ratio is nearly zero for a large portion of the experiment. This corresponds to the experiment being dominated by radiation. For this reason the radiation-optimal experiment was to heat the sample for 30 
seconds and then data was sampled for 100 seconds. Again a longer sampling time was used than the $D$ value indicated was optimum.

Simulated experiment 3 consisted of the conduction-optimal and radiation-optimal heating events combined together. The residuals for simulated experiment 3 are given in Figure 9. The estimated parameters and their $90 \%$ confidence intervals are given in Table 5 . As can be seen in the figure and the table, the temperatures and the parameters are estimated accurately.

Table 5: Parameter values for combined conduction and radiation experiments

\begin{tabular}{cccccc}
\hline \hline & & & & & \\
& $e_{0}$ & $e_{1}$ & $\omega$ & $\mathrm{F}$ & $\mathrm{a}$ \\
\hline Exact & $9.850 \mathrm{E}+00$ & $-2.630 \mathrm{E}-03$ & $9.930 \mathrm{E}-01$ & $6.850 \mathrm{E}-03$ & $3.890 \mathrm{E}+02$ \\
\hline Estimated & $9.844 \mathrm{E}+00$ & $2.626 \mathrm{E}-03$ & $9.930 \mathrm{E}-01$ & $6.838 \mathrm{E}-03$ & $3.905 \mathrm{E}+02$ \\
\hline Percent Error & $6.271 \mathrm{E}-02$ & $1.666 \mathrm{E}-01$ & $-2.696 \mathrm{E}-05$ & $1.792 \mathrm{E}-01$ & $-3.960 \mathrm{E}-01$ \\
\hline $90 \%$ Confidence Interval & $1.944 \mathrm{E}-02$ & $1.668 \mathrm{E}-05$ & $2.818 \mathrm{E}-05$ & $9.107 \mathrm{E}-06$ & $8.771 \mathrm{E}-01$ \\
\hline \hline
\end{tabular}

The sensitivity coefficients (normalized by their maximum value) are plotted for simulated experiment 1 in Figure 10. The sensitivity for $a$ has nearly the same shape as the sensitivity for $F$. This indicates the sensitivities are almost linearly independent and is probably the reason it is hard to determine the value for $a$. A different choice of conduction parameters could probably be found that could be determined more accurately from simulated or actual experiments.

Simulated experiment 3 provides more accurate results than either experiment 1 or experiment 2. For all the experiments, the strict use of the normalization of $D$ by the number of samples taken tended to end the experiments before all useful data was taken. Unnormalized values of $X^{T} X$ values would indicate the sample should be heated to steady-state and then cooled back to room temperature, which would require a very long experiment. The best data duration appears to be somewhere between the duration determined by strict use of the maximum value of the normalized and unnormalized values of $X^{T} X$.

\section{Conclusion}

For future use of high porosity materials in thermal protection systems for reusable launch vehicles, their performance needs to be characterized over a wide range of temperatures and pressures. The goal of this research is to develop general-purpose protocols for designing transient experiments to measure thermal properties of high porosity materials. As a first step, a metal foam material was studied with an experimentally validated thermal model.

The $D$ optimality criterion and consideration of the heat fluxes can lead to suitable experiments for determining the properties. Maximization of the optimality criterion $D$ lead to an experiment containing one heating event that could be used to determine the radiation and conduction parameters. Strict use of the $D$ parameter leads to experiments that were too short in the sense that more accurate parameter estimates could be obtained from simulated data sets of longer duration. By considering the model itself and in particular its conduction and radiation behavior, a set of two shorter heating events were found that provided more accurate parameter estimates.

The radiation parameter $\omega$ was easily found to a high degree of accuracy by all of the experiments. The coupling coefficient, $a$, was the hardest parameter to determine. The sensitivity of the of the coupling coefficient and the sensitivity of the solid conduction efficiency, $F$, are similar in shape which indicates they are close to linearly dependent. The coupling coefficient was an ad-hoc parameter added to Sullins' model in order to obtain agreement with experimental data at high pressures. This suggests that there may be a different pair of conduction parameters that could adequately model the conduction process and provide more robust estimation than exhibited here.

\section{Acknowledgement}

This work was supported by NASA-Langley grant NAG-1-01087 under the supervision of Max Blosser. 


\section{Appendix}

The temperature dependent properties for nitrogen are from Daryabeigi. ${ }^{12}$

\subsection{Temperature Dependent Nitrogen Properties}

$$
\begin{aligned}
\rho_{g} & =-1.734 E-4+342.216 / T \\
c_{g} & =1.083 .545-0.328 T+6.949 E-4 T^{2}-2.82 E-7 T^{3} \\
k_{g} & =2.048 E-3+8.751 E-5 T-2.462 E-8 T^{2} \\
P r & =0.854-7.085 E-4 T+9.008 E-7 T^{2}-3.207 E-10 T^{3}
\end{aligned}
$$

\section{References}

1 Dorsey, J. T. et al., "Metallic Thermal Protection System Technology Development: Concepts, Requirements and Assessment Overview", Proceedings, 40th Aerospace Sciences Meeting, Jan 14-17, 2002, Reno, Nevada, paper AIAA 2002-0502.

2 Beck, J. V. and Arnold, K. J., Parameter Estimation, 1977, Wiley, NY.

3 Taktak, R., Beck, J. V., and Scott, E. P., "Optimal experimental design for estimating thermal properties of composite materials," International Journal of Heat and Mass Transfer, Vol. 36, No. 12, 1993, pp. 2977-2986.

4 Dowding, K. J. and Blackwell, B. F., "Sensitivity Amalysis for Nonlinear Heat Conduction," Journal Heat Transfer, 123, 1-10 (2001).

5 Cole, K. D., "Thermal Characterization of Functionally Graded Materials-Design of Optimal Experiments," 8th Joint AIAA/ASME Thermophysics and Heat Transfer Conference, St. Louis, MO, June 24-27, 2002, paper AIAA 20002-2882.

6 Cole, K. D., "Analysis of Photothermal Characterization of Layered Materials-Design of Optimal Experiments," 15th Symposium on Thermophysical Properties, Boulder, CO, June 22-27, 2003.

7 Daryabeigi, K., "Heat Transfer in High-Temperature Fibrous Insulation," AIAA J. Thermophysics and Heat Transfer, vol. 17, no. 1, 2003, pp. 10-20.

8 Bhattacharyya, R., "Heat Transfer Model for Fibrous Insulations," Thermal Insulation Performance, D. L. McElroy and R. P Tye, editors, American Society for Testing and Materials Technical Publication 718, Philadelphia, pp. 272-286, (1980).

9 Yuen, W. W., Takara, E., and Cunnington, G., "Combined Conductive/Radiative Heat Transfer in High Porosity Fibrous Insulation Materials: Theory and Experiment," Proceedings, 6th ASME-JSME Joint Thermal Engineering Conference, March 16-20, 2003, paper TED-AJ03-126.

10 Zhu H., et. al "Minumum Mass Design of Insulation Made from Functionally Graded Design", Proceedings, 43rd AIAA Structures, Structural Dynamics, and Materials Conference, Denver CO, April 22-25, 2002, paper AIAA-2002-1425.

11 Sullins, A. D., and Daryabeigi, K., "Effective Thermal Conductivity of High Porosity Open Cell Nickel Foam, "Proceeding of the 35th AIAA Thermophysics Conference, Anaheim CA, June 11-14, 2001, paper AIAA 2001-2819.

12 Daryabeigi, K., Design of High Temperature Multilayer Insulation for Reusable Launch Vehicles, Ph.D. dissertation, University of Virginia, May 2000. 


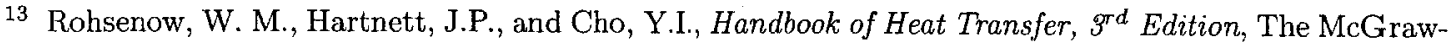
Hill Companies, Inc., 1998.

14 Daryabeigi, K., Knutson, J. R., and Sikora, J. G., "Thermal Vacuum Facility for Testing Thermal Protection Systems," NASA TM-2002-211734, June 2002.

15 Press, W. H., Numerical Recipes in FORTRAN 77, Cambridge University Press, New York, 1996.

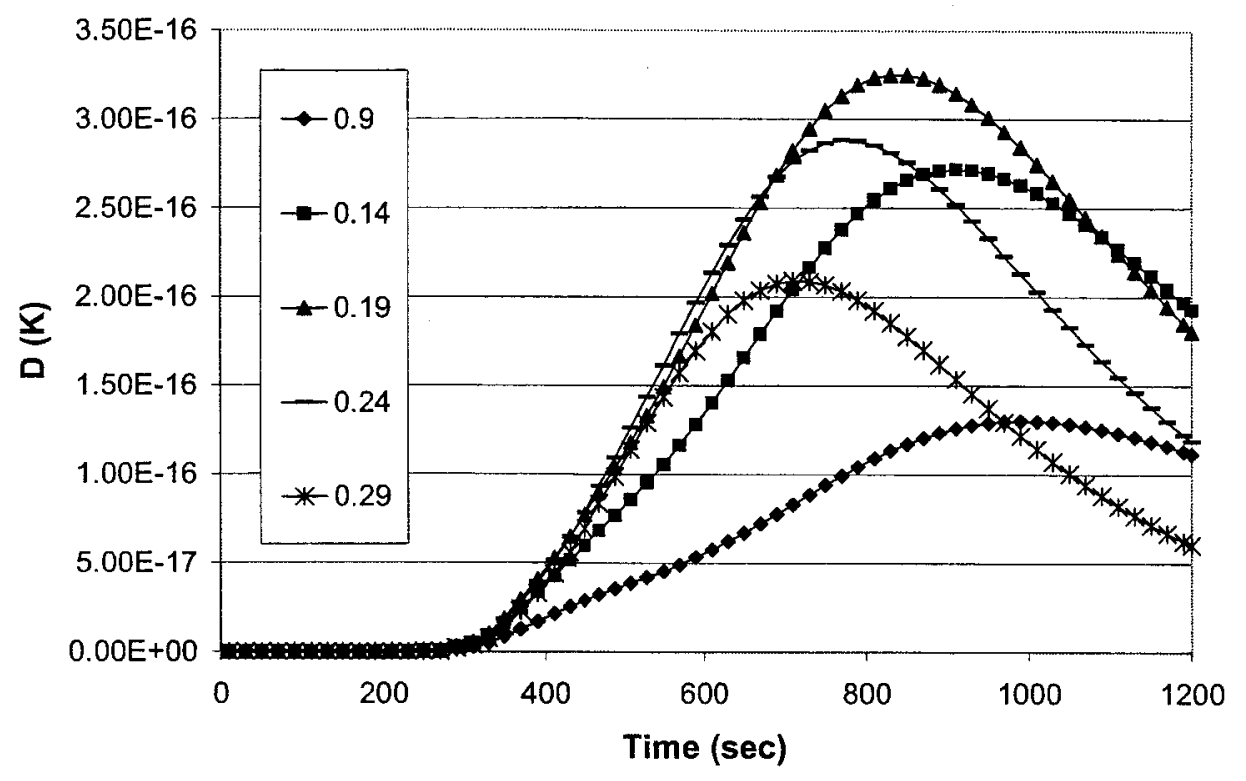

Figure 1: D for 269 seconds of heating at various pressures in $\mathrm{mm} \mathrm{Hg}$ 


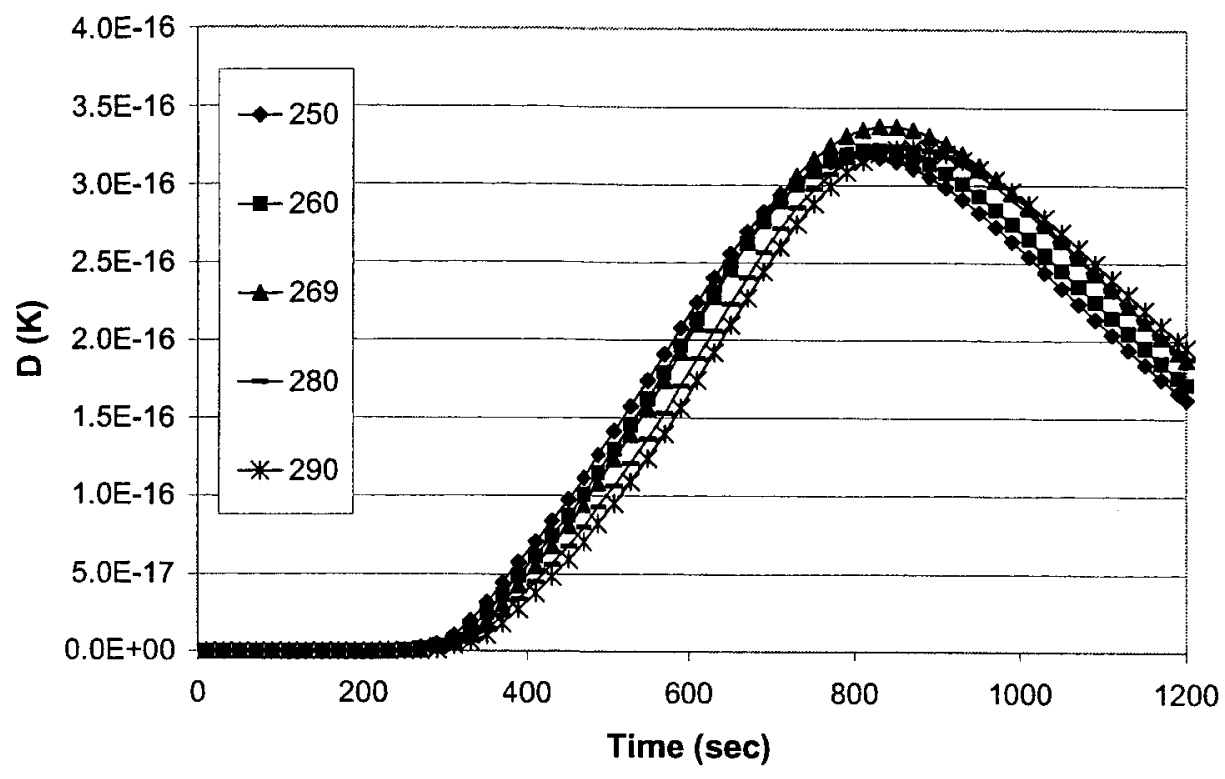

Figure 2: $\mathrm{D}$ for $\mathrm{P}=0.19 \mathrm{~mm} \mathrm{Hg}$ and various heating times

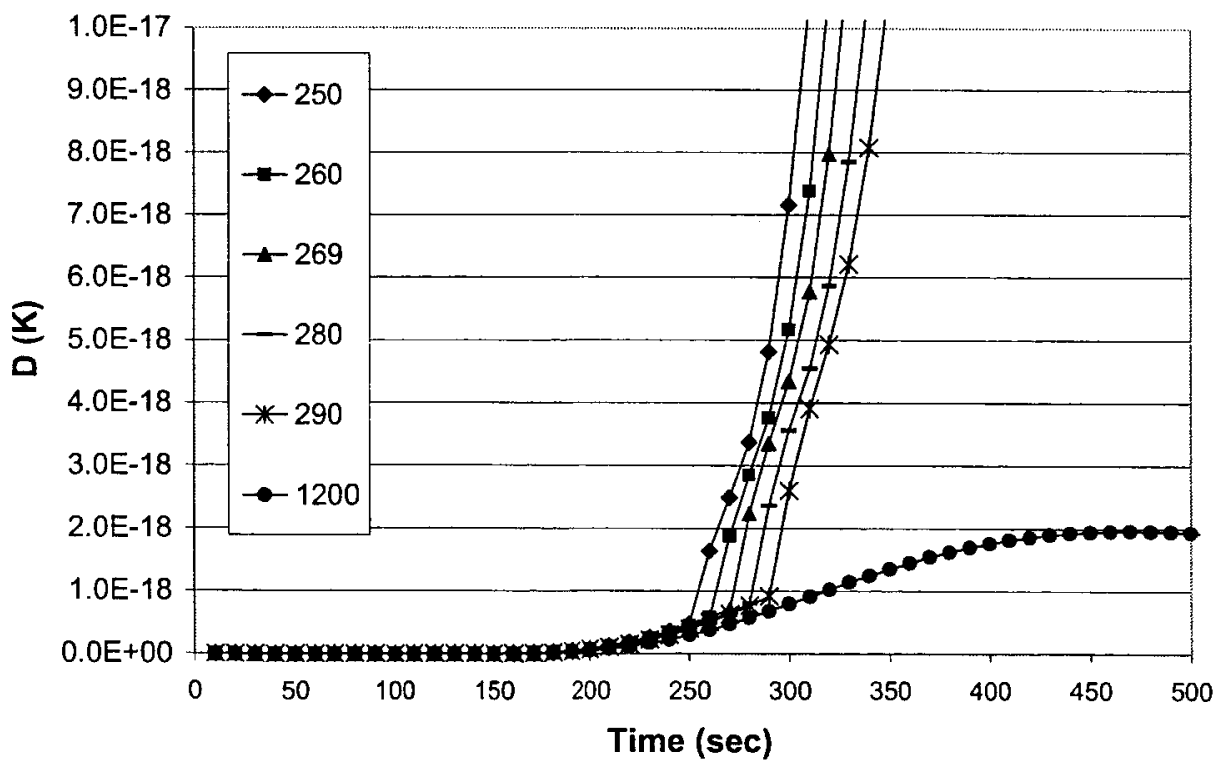

Figure 3: $\mathrm{D}$ for $\mathrm{P}=0.19 \mathrm{~mm} \mathrm{Hg}$ during heating. 


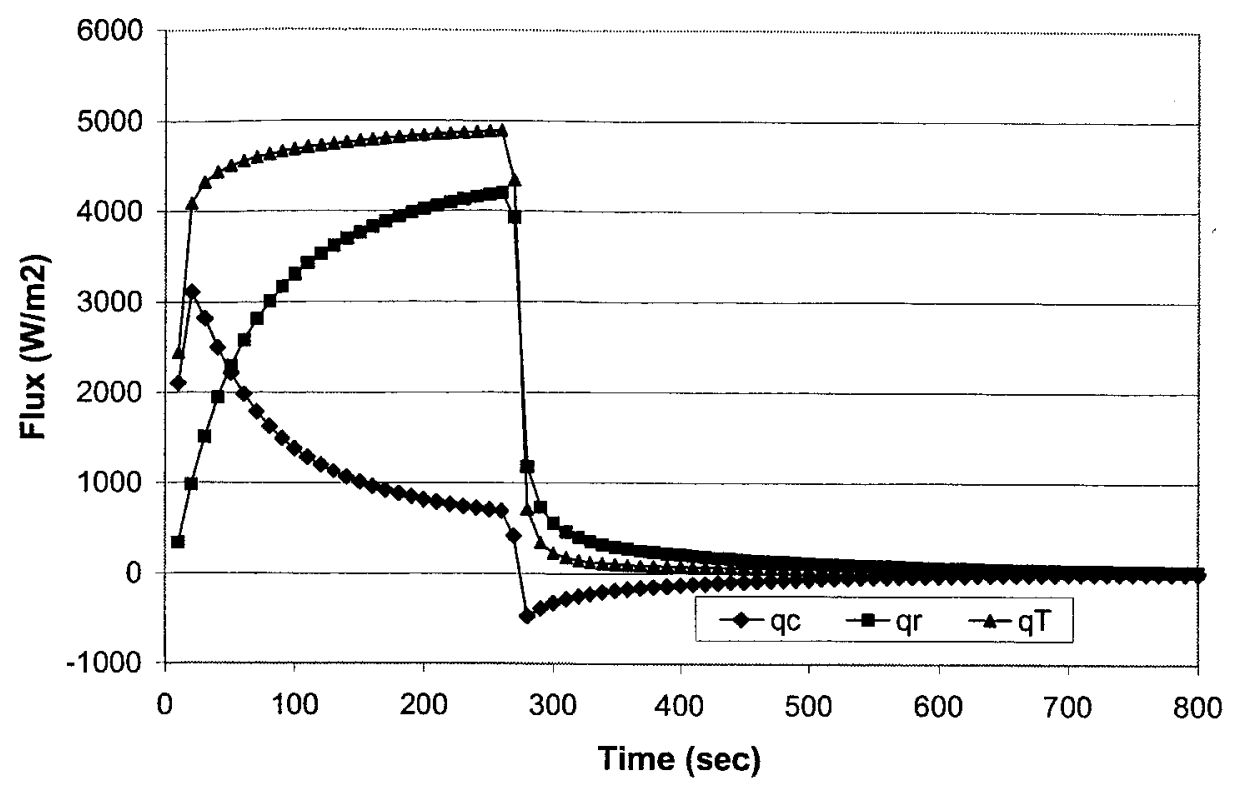

Figure 4: Heat fluxes for $\mathrm{P}=0.19 \mathrm{~mm} \mathrm{Hg}$ and 280 seconds of heating.

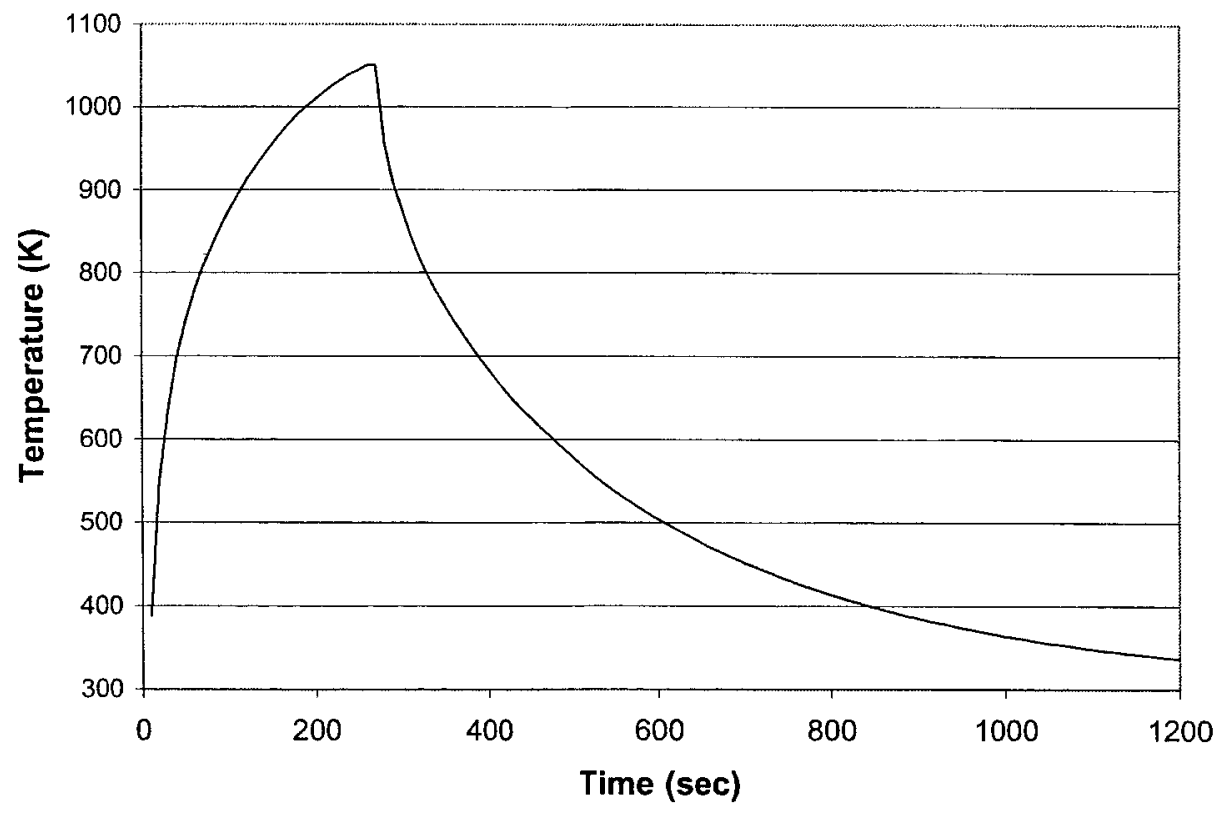

Figure 5: Temperature for $\mathrm{P}=0.2 \mathrm{~mm} \mathrm{Hg}$ and 280 seconds of heating. 


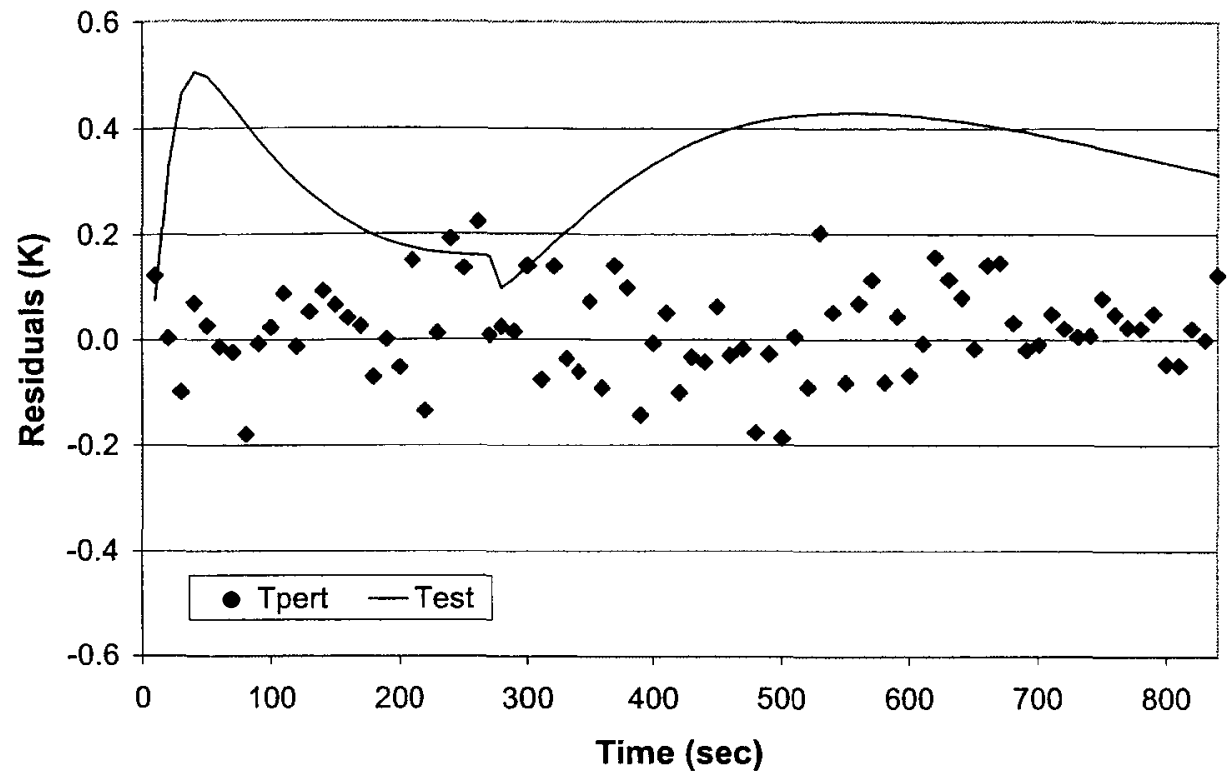

Figure 6: Temperature residuals for simulated experiment 1.

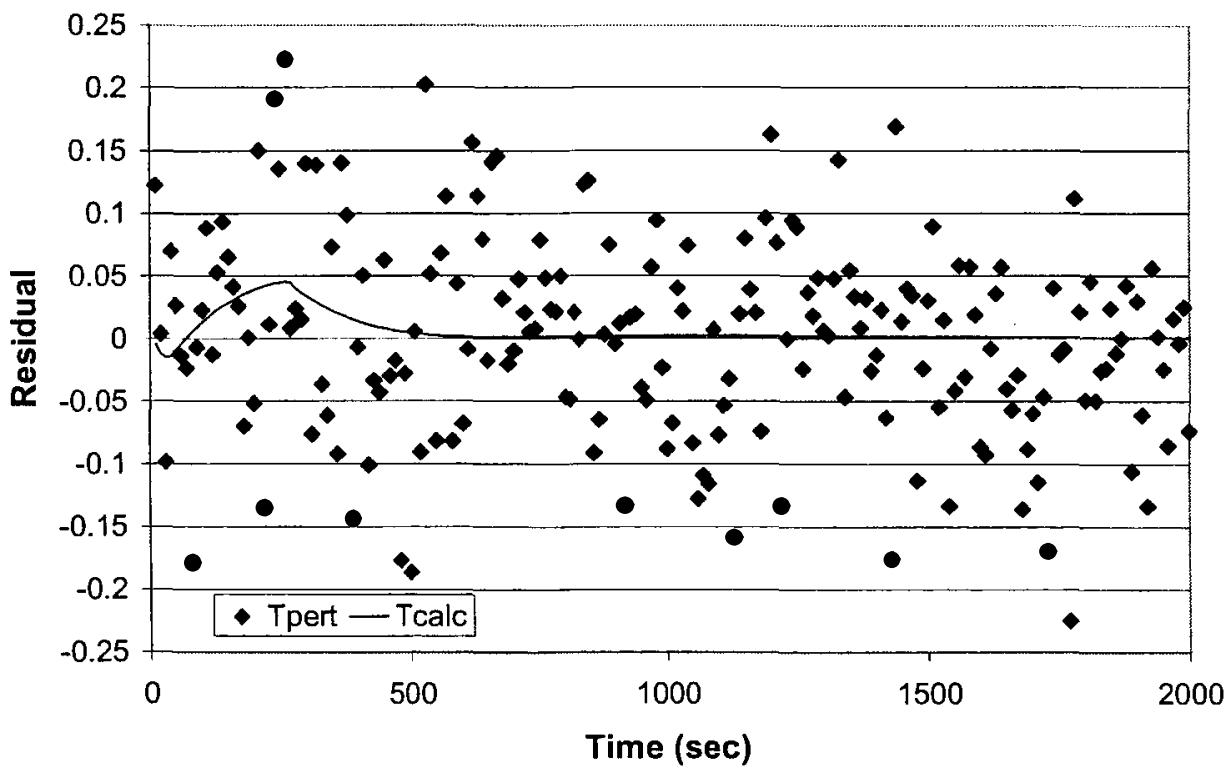

Figure 7: Temperature residuals for simulated experiment 2. 


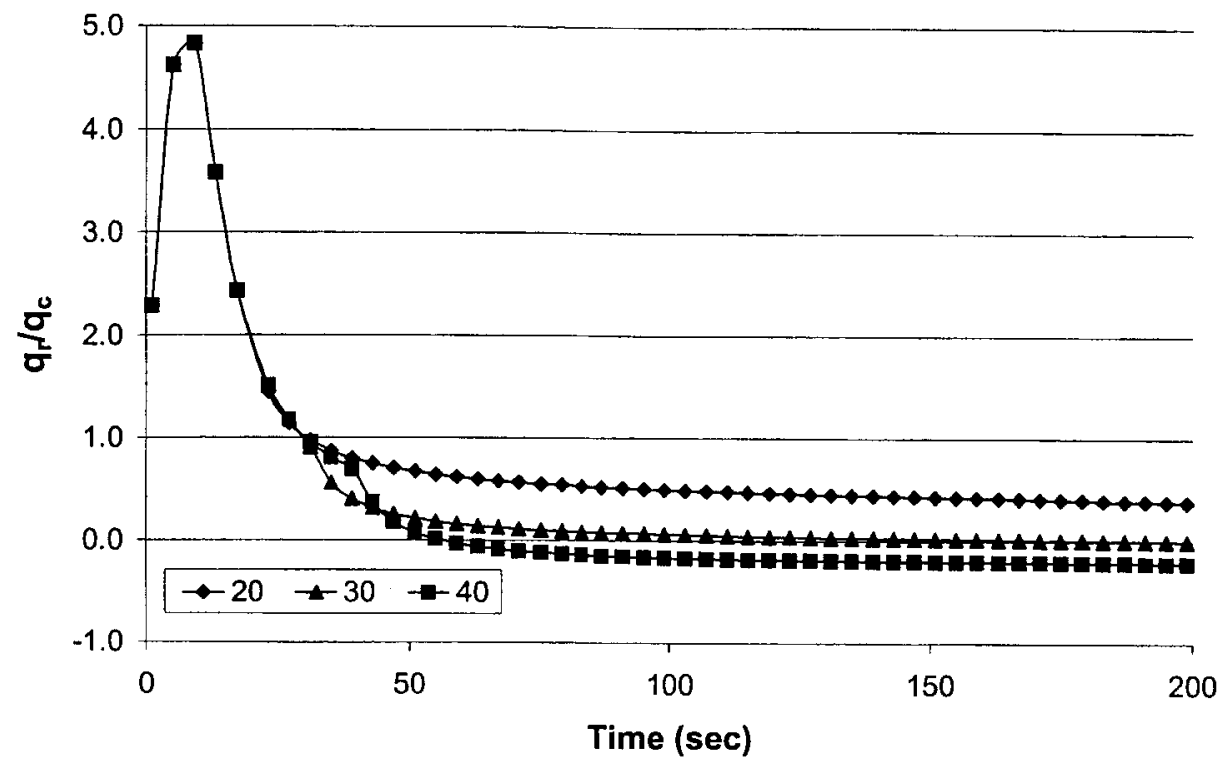

Figure 8: Ratio of conduction to radiation flux for different heating times in seconds.

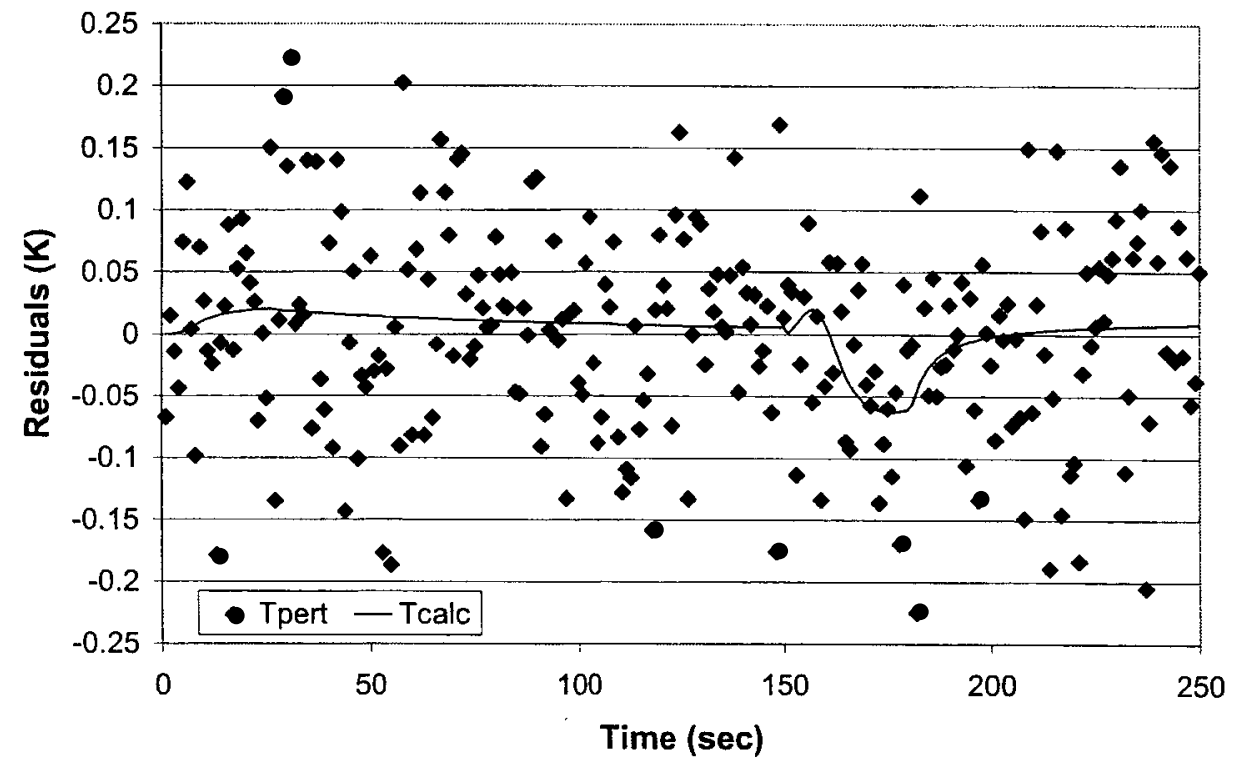

Figure 9: Temperature residuals for simulated experiment 3. 


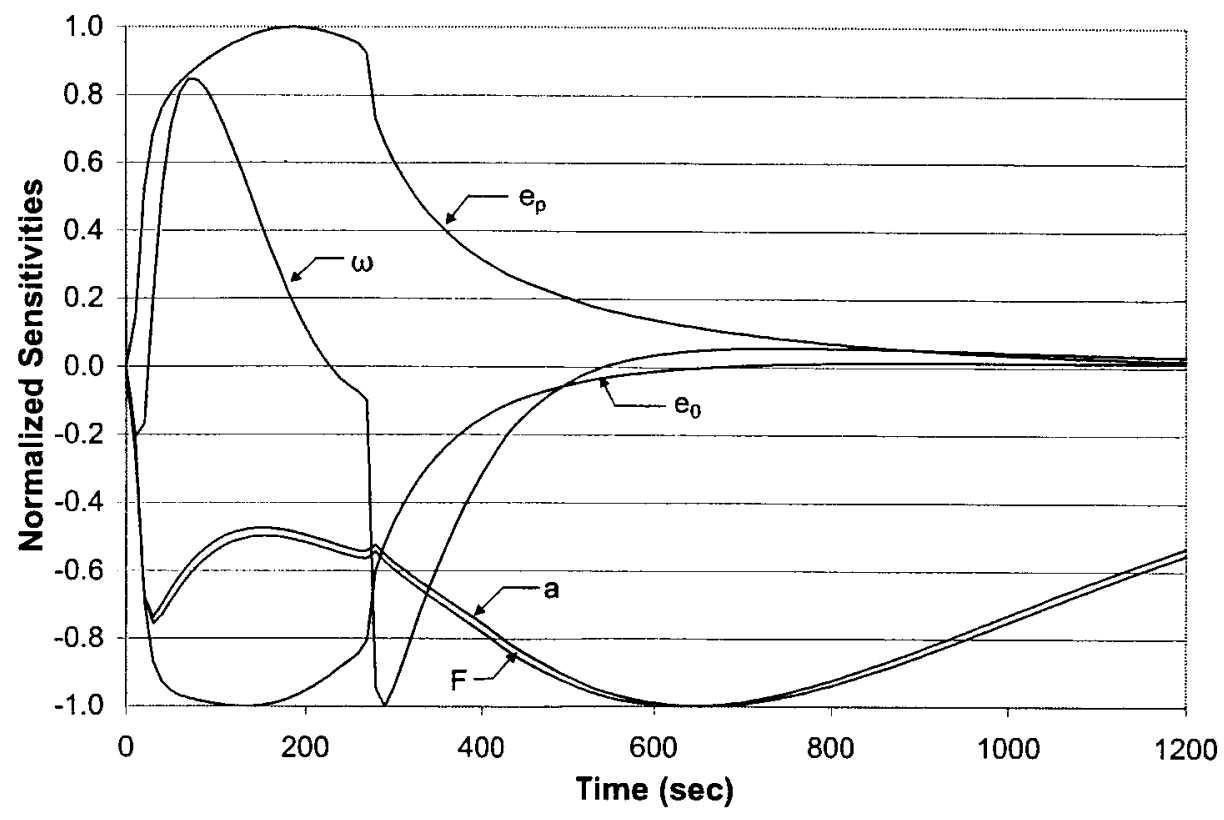

Figure 10: Sensitivities for conduction portion of simulated experiment 3 